\title{
Teaching Strategies during the Covid-19 Pandemic - A Review
}

\author{
Deepa Jatti Patil ${ }^{1}$ \\ ${ }^{1}$ Department of Oral Medicine and Radiology, K. M. Shah Dental College and Hospital, Sumandeep \\ Vidyapeeth (Deemed to Be University), Piparia, Vadodara, Gujarat, India.
}

\section{ABSTRACT}

The Covid pandemic transformed the teaching methodologies in dental education. The classes had to be conducted online and teaching methods had to be modified to suit the dental curriculum. Novel teaching methodologies were devised following the basic principles of Blooms taxonomy. The teaching methodologies have to be effective and incorporate the latest information technology methods. The advancements in technology and availability of internet resources have facilitated online learning. Various platforms are available for teaching and disseminating knowledge. Learning has transformed from the traditional Didactic teaching to flipped class rooms and blended learning. As the pandemic is progressing there is deficiency of health care workers and resources. In spite of this the medical teaching has to continue to fill the gap. Oral Medicine and Radiology is an important subject in the dental curriculum and a bridge between medicine and dentistry. This manuscript reviews the various teaching methodologies employed in the Oral Medicine and Radiology curriculum during this pandemic. This review also highlights the importance of technology and simulation-based learning to enhance the clinical skills of the students.

\section{KEY WORDS}

Covid Pandemic, Online Learning, Flipped Classroom, Information Communication Technology, Blended Learning, Online Patient Simulation, Adaptive Tutorials
Corresponding Author: Dr. Deepa Jatti Patil, Associate Professor, Department of Oral Medicine and Radiology, K. M. Shah Dental College and Hospital, Sumandeep Vidyapeeth (Deemed to Be University), Piparia,

Vadodara, Gujarat, India.

E-mail: iafdeepa@gmail.com

DOI: $10.14260 / \mathrm{jemds} / 2021 / 510$

How to Cite This Article:

Patil DJ. Teaching strategies during the covid-19 pandemic - a review. J Evolution Med Dent Sci 2021;10(31):2494-2498, DOI: $10.14260 / \mathrm{jemds} / 2021 / 510$

Submission 14-04-2021, Peer Review 02-06-2021, Acceptance 05-06-2021, Published 02-08-2021.

Copyright (c) 2021 Deepa Jatti Patil. This is an open access article distributed under Creative Commons Attribution License [Attribution 4.0 International (CC BY 4.0)] 


\section{BACKGROUND}

The Covid-19 pandemic caught us unawares and changed our life within a span of few days. Most of us had to face numerous challenges, both on the professional as well as domestic front. All aspects of human life, including education were disrupted. Students vanished from the colleges and patients from the clinics. In spite of all this, the learning process had to continue and students had to be engaged in their curriculum. The traditional face to face learning has been transformed to online learning. However, online learning comes with its own challenges. It has to encompass all facets of classroom learning like being effective, innovative and invoke interest in the subject. At this time of pandemic other non-medical professional courses are continuing online. Medical and dental education involves learning through patient interaction and communication. So, can we justify the patient learning by teaching students online? Certainly, it can be done to a greater extent as the teaching has to continue as in future there can be a paucity of health care workers.

Today is the world of digital culture incorporating information and communication technology (ICT). ICT can complement, enrich and transform education for the better. ICT tools help to communicate, create, disseminate, store, and manage information. Today is an era of digital literacy, including the skills of searching and producing information and has thus become an important deliberation for curriculum frameworks. ICT has revolutionised the teaching and learning process and has found its full use during this pandemic. ${ }^{1}$ The UNESCO has defined ICT as "ICT is a scientific, technological and engineering discipline and management technique used in handling information, its application and association with social, economic and cultural matters" ICT has revolutionised the teaching methodologies and has brought about several changes in the education system. It is beneficial to both teachers and students providing a common platform for sharing knowledge as well as learning.

The teaching methodologies have to be based on Bloom's Taxonomy of Educational Objectives thus maximizing educational value. It is imperative that the student learning be based on knowledge-based, skill-based, and affective goals. The students should progress from gaining knowledge, application to higher components of analysis, synthesis, and evaluation. Using different online teaching methodologies, the students can acquire high order thinking skills to analyse, interpret and apply the knowledge gained during the learning process. $^{2}$ We will discuss the various techniques for effective teaching during the pandemic situation following the educational objectives and incorporating new technologies.

\section{ONLINE TEACHING}

\section{Principles of Online Learning}

According to Levin et al. ${ }^{3}$ there are five important characteristics to enhance the online learning. This consists of pertinent and thought-provoking projects, harmonized learning settings, suitable and judicious response from instructors, fostering peer learning, and flexibility in the teaching and learning methodology. The online teaching setting must help the students to critically analyse, interact with the peers and teachers, contend, explore, create and share new ideas. The online courses can be classified into synchronous and asynchronous learning. In synchronous learning the teacher and students are online and the knowledge transfer occurs at the same time. In asynchronous learning the teaching modules are placed on the web-based portals and students can login and access the modules. ${ }^{4}$

\section{General Outline of Online Teaching}

There is a contrast in the teaching methodology followed during virtual and in person class lectures. Classroom teaching lacks out of box thinking. Thought provoking questions during the class and student feedback can control student behaviour and enhance the capacity of online classes. Teaching staff should devise creative learning material, and assign students with more innovative and practical work like case studies. With these measures the aptitude of students can be improved with more active participation during online learning. ${ }^{5}$ Prior to the online session time, pre-class specific reading material should be provided. A brief explanation of online content understood by the students can improve online class discussion.

\section{Framing Learning objectives}

To improve understanding and for effective communication it is important to enlist the learning objectives. The learning objectives should be student centred and focus on their behaviour and ability to absorb knowledge and interpret the learning material provided to them. The modules should be designed in a step wise manner, from basics to high ordered thinking. For example, for the undergraduates posted in the Oral Radiology department the basics should include identifying anatomical landmarks, X-ray production, and projecting intra-oral radiographs in the third year. In the final year they can be taught about radiographic interpretation of pathologies and projecting and interpreting extra-oral radiographs. Principles and interpretation of Cone beam computed tomography (CECT), computed tomography (CT) and Magnetic resonance imaging (MRI) are included in the post graduate curriculum.

\section{Requirements for Online Teaching}

- Establishing a common platform for effective communication between the students and teachers, is vital. Google classroom is a useful tool which can be used to impart knowledge, convey information, and upload digital content for students in the form of videos, patient cases and radiographs for practice exercises.

- Comprehensive lesson plans with teaching aids like e content presentations.

- Make contingency plan for emergency problems due to network and server errors.

- Modular Teaching - Divide the teaching content into smaller units. As students lack focus for a longer span, the modular teaching method is most appropriate. The teaching program should be split into small sessions of shorter duration of not more than half an hour.

- "Voice" of the teacher should be emphasised. During offline teaching a teacher's body language and facial expression are important teaching tools. During a virtual 
class voice modulation is imperative so that the students can grasp the key points.

- Creating E content - The curriculum can be made interesting and easy to understand by creating E content. It can be in the form of slide cast and screen cast videos, table top videos and pod cast.

Table top videos of Intraoral and Extra oral techniques, principles of projection geometry can be made and uploaded. The table top and screen cast videos on various topics were created by our department and students found them useful and interesting.

\section{Platforms for Online Teaching ${ }^{5}$}

1. Cloud computing platforms like Zoom, G-suite, Microsoft teams, Webex which allow video conferencing.

2. TUSK platform contains various syllabi, course work, schedules, assessment patterns, multiple choice questions, tips to solve clinical Scenarios-This is being followed by Christian Medical College.

3. LISTSERV is an online google group used by FAIMER Fellowship programmes.

4. Telemedicine software system.

5. EDUSAT is the first satellite system for delivering distance education.

\section{DIFFERENT TEACHING METHODOLOGIES}

\section{Didactic Teaching}

This form of teaching focusses on the instructor's ability to teach and explain the content. The knowledge and content of the lecture is not under scrutiny. The instructor lectures and the students do not actively participate as they are passive listeners. The students have to listen attentively and memorize the study material. Contemporarily this methodology is a form of didactic teaching. ${ }^{6}$

\section{Limitations}

This form of learning is a monologue process, limiting student interaction and motivation. This form of teaching is particularly unsuitable in online teaching. To enhance student interaction, other teaching methodologies have been devised and implemented.

\section{Flipped Classroom}

Flipped classroom is a pedagogical approach in which direct instruction moves from the group learning space to the individual learning space, and the resulting group space is transformed into a dynamic, interactive learning environment where the educator guides students as they apply concepts and engage creatively in the subject matter. ${ }^{7}$ During classroom learning, the students listen passively, try to understand the teaching content whereas the higher order thinking is usually done outdoors. In case of the flipped classroom model, the learning is flipped. The students can finish the lower level of cognitive work like memorising and understanding prior to the lecture. In the classroom they can discuss higher cognitive levels of learning with both their peers and teachers. During class they can apply and analyse and after class they can evaluate and create.
For example, screencast video can be made of a particular topic like "Principles of radiographic Interpretation" and uploaded. This video can depict methods of interpretation of pulp and periapical lesions, cysts, periodontal diseases etc. Later the students can be divided into groups and each student can be assigned a radiograph to interpret and diagnose. In the next session each student can discuss their individual interpretation with their peers and carry out in-depth discussion about the topic. Similarly, different topics of pathogenesis of oral cancer, oral submucous fibrosis (OSMF), and oral lichen planus can be first taught in a screen cast video with Mind mapping technique and then students can prepare them on their own and explain to the entire class. Prescription writing: A video of detailed prescription writing technique can be uploaded to the students. The students can then be assigned to write prescription for dentoalveolar abscess, TMJ pain and so on. The individual prescriptions can be discussed in the class and doubts can be cleared. This will invoke interest in the students.

\section{Blended / Hybrid Learning}

This form of teaching involves both offline and online content. This methodology involves an instructor delivering real-time instructions in the classroom and to remote students at the same time. This teaching methodology includes e learning aids as classified below. ${ }^{8}$

- Video conferencing

- Learning management systems

- Online exercises

- Online discussion forums

- Pre-recorded video instruction.

Presently, as the students are at home, the various intra oral and extraoral techniques can be taught online using table top videos and exercises can be given to write and draw the principles of different techniques. When the students join the offline classes, they can start practising the technique on patients.

\section{Case Based Learning (CBL)}

CBL focusses on preparing students for clinical practice by the medium of demonstrating the patients' lesions and conditions. It is a form of learning associated with the theoretical knowledge on patients with the help of "inquiry-based learning methods". A discussion of the case taught can help the students to self-assess, understand and apply a high order of cognition. This learning methodology fosters active participation with a productive outcome. ${ }^{9}$ Case scenarios During this pandemic, students are unable to attend clinics and examine patients. In order to effectively teach them about patient diagnosis and management, the CBL technique can be adopted and was followed in our department. For example, during teaching the various potentially malignant disorders, individual cases of leukoplakia or OSMF can be discussed in detail by showing the pictures of various lesions, discussing the aetiology and deriving a treatment plan for the cases.

The students can be subdivided into groups and case discussions can be done. Then, each student will be given practice exercises on oral mucosal lesions and asked to write down the description of the lesion, diagnosis, differential 
diagnosis, investigations, and treatment plan for the case. CBL goes beyond simple identification of correct answers and is more aligned with either evidence of critical thinking or changes in behaviour and generalizability of learning new cases.

\section{Sandwich Principle of Learning}

This methodology incorporates the sandwich principle of individual learning and personal belongings \& consecutively alternates between individual and collective learning phases. Collective learning phases are passive. This method of teaching is student centred and focusses on the student's ability to learn. The student behaviour and concentration span are taken in to consideration. Traditionally, during a lecture the students are passive listeners. In this technique, during the collective learning phase a concise lecture is delivered in a short duration of 20 to $25 \mathrm{~min}$, which is within the attention span of the students. The individual learning phases are active learning phases wherein the students replicate, analyse and understand the content delivered. Various projects, group discussions and peer learning can activate this process. The knowledge learnt can be applied and helps in long-term retention of the content. This interchange of collective and individual learning phases, involves a switch between passive and active learning. ${ }^{10}$

This form of learning was used in our department. After the online lectures the topics were divided among students for peer teaching. The students were divided into different batches. Each batch comprised of four students and one topic was divided among them. The students learned and comprehended the topic better and cleared their doubts. Written assignments were also given to the students for testing their understanding of the topic. Assignment of multiple-choice questions after lecture is also a good way of getting student feedback and was followed regularly by our department.

\section{Online Patient Simulations}

Developing clinical reasoning skills is an essential part in the diagnostic work up process. Advanced tools are required to train the student to achieve the reasoning skills. Online patient simulations (OPS) are computer-based programs, replicating real-life clinical scenarios and assist in teaching reasoning skills. Various learning models propose that multiple experiences to a greater variety of clinical cases through simulations can enhance the cognition by reorganization and constructing complex intellectual illustrations. This type of learning by experience enables reflection, which helps the students to retain skills. OPS can be combined with classroom teaching and allows students to collect data and make diagnoses without troubling patients. The logical advantages include low cost, wide exposure, can be performed virtually and customised to the learner with latest knowledge. 11

General physical examination is a vital part of our curriculum for undergraduates and post graduates. OPS can be used for teaching students to check vital signs, pallor, icterus, cyanosis, clubbing, oedema and lymphadenopathy. The clinical skills of students can be enhanced to diagnose anaemia, thyroid disorders, cardiovascular diseases, endocrine disorders, respiratory disorders etc. Clinical tips to diagnose systemic disorders through oral manifestations can be performed through OPS.

Videos of patients explaining their clinical situations can be displayed to the students. Clinical vignettes and characteristic features can be described in the simulations. The students have to note down important questions and arrive at a diagnosis along with differential diagnosis.

\section{Adaptive Tutorials for Radiology}

This is a type of virtual intellectual training technique, allowing asynchronous e-learning. These radiology tutorials deliver pre-recorded guided information with quick customised feedback. To evade cognitive overload, the depth of information in the adaptive tutorials is aimed specifically at students' level of understanding. In addition, the module is subdivided into practical sessions and to introduce the complex concepts in phases with suitable feedback. These tutorials can be used for interpreting images of CBCT, CT and MRI. These engaging, interactive tutorials offer better understanding when compared with the peer reviewed webbased resources (Radiopaedia). Previous studies have depicted better performance by undergraduate students exposed to adoptive radiology tutorials in terms of objective measures of learning like information of indications and interpretations of basic imaging as compared to their peers who received knowledge from web-based resources. ${ }^{12}$ The adaptive tutorial can include the following teaching modules

1. Types of radiation

2. Sources of natural and background radiation

3. Radiation exposures from different imaging techniques

4. Enlisting the various imaging modalities

5. Basics of image interpretation with a checklist of the points to be covered

6. Imaging modalities for hard and soft tissue imaging

7. Enumerating various clinical scenarios- maxillofacial trauma, oral cancer and indications of the imaging technique in such situations

8. Identification of normal anatomical landmarks and pathological conditions on the radiographic images.

9. After the basic imaging procedures are covered advanced software for fusion imaging, three-dimensional reconstruction can be incorporated.

The learning material should be disseminated incrementally daily and multiple-choice questions to be incorporated at the end of the module to assess the student learning. Feedback should be taken at each step to improve the learning outcome.

The tutorial should be for a span of 2-3 months with an examination at the end of module for grading the students.

\section{Smart-Phone Based Learning 13}

In this form of learning mobile devices are used for sharing the learning content through social interaction. Among all the elearning tools mobiles are most popular as they are easy to use, accessible, economical and ease of internet connectivity. They are portable and the learning environment can be easily modified. There is a sudden surge in popularity of using the mobiles as a learning tool in medical and dental curriculum. ${ }^{13}$ In case of oral radiology this medium of learning can be used to teach complicated chapters which cannot be covered in 
online lectures. This includes tracing of anatomical landmarks on extra-oral radiographs, assessing the skeletal deformities, identification of syndromic patients with peculiar radiographic features and estimating age through dental radiographs.

Smart phones can also be used to learn drug dosage calculation, assessment of patients with high risk of certain disorders like sleep apnoea, assessment of tobacco addiction, questionnaires to assess depression and anxiety, assessment of pain scales etc. through various apps like Docplexus and Curofy which facilitate learning through clinical case scenarios followed by a quiz. Webinars and webcast by governing bodies of speciality can help in learning and dissemination of knowledge. Searching various databases like PubMed, Scopus and Cochrane can be easily performed to access the medical literature.

\section{Objective Structured Clinical \& Practical Examination}

It's an approach to assess the clinical competence of students in a structured format using different stations. This includes the objective structured practical and clinical examination. (OSPE) This type of examination assesses the three learning domains i.e., cognitive, affective and psychomotor. These domains are not evaluated in the traditional examination pattern. This type of examination assesses the clinical application of knowledge. It can be used to assess the clinical skill of the student in a short span. OSPE is a reliable, valid and objective tool to assess the student's skill and knowledge application. Although this type of examination is time consuming, the traditional examination methods need to replaced by newer examination methods. This form of clinical assessment is gaining popularity and many universities are incorporating this form of examination in their curriculum. ${ }^{14}$ We conducted online practical examinations with this approach and were quite successful in assessing the students.

\section{CONCLUSIONS}

We are facing numerous challenges during this pandemic. Deficiency of health care workers and resources is a matter of concern. Postgraduate students and interns are being utilized for Covid duties. At this time the undergraduate teaching has to continue to fill the gap and provide manpower. This pandemic has transformed education with innovative strategies to involve and engross students. There is uncertainty as to when the formal classes would resume. Its time to embrace technology and innovate teaching methodologies for effective teaching and learning. It's imperative to teach the students and at the same time keep them focused and make the classes interesting and creative. Various forms of learning can be incorporated and inculcated according to the desired curriculum. In the future the blended form of learning will gain popularity over the other teaching methodologies as the situation demands. Careful planning and implementation with active participation both by the teachers and students is the key to success of teaching during this pandemic.
Financial or other competing interests: None.

Disclosure forms provided by the authors are available with the full text of this article at jemds.com.

\section{REFERENCES}

[1] Bao W. COVID-19 and online teaching in higher education: a case study of Pekiang University. Human Behaviour and Emerging Technologies 2020;2(2):113-5.

[2] Anderson LW, Krathwohl DR, Bloom BA. A taxonomy for learning, teaching and assessing: a revision of Bloom's taxonomy of educational objectives. Complete ed. New York: Longman 2001.

[3] Levin SR, Waddoups GL, Levin J, et al. Highly interactive and effective online learning environments for teacher professional development. International Journal of Educational Technology 2000;2:1-13.

[4] Tallent-Runnels MK, Thomas JL, Lan WY, et al. Teaching courses online: a review of the research. Review of Educational Research 2006;76(1):93-135.

[5] Sahi PK, Mishra D, Singh T. Medical education amid the COVID-19 pandemic. Indian Pediatr 2020;57(7):652-7.

[6] Austin S. Didactic Approaches. In: Volkmar FR, ed. Encyclopedia of autism spectrum disorders. New York, NY: Springer 2013.

[7] Chen F, Lui AM, Martinelli SM. A systematic review of the effectiveness of flipped classrooms in medical education. Med Educ 2017;51(6):585-97.

[8] Westerlaken M, Christiaans-Dingelhoff I, Filius RM, et al. Blended learning for postgraduates; an interactive experience. BMC Med Educ 2019;19(1):289.

[9] McLean SF. Case-based learning and its application in medical and health-care fields: a review of worldwide literature. J Med Educ Curric Dev 2016;3:JMECD.S20377.

[10] Bock A, Idzko-Siekermann B, Lemos M, et al. The sandwich principle: assessing the didactic effect in lectures on cleft lips and palates. BMC Med Educ 2020;20(1):310.

[11] Plackett R, Kassianos AP, Kambouri M, et al. Online patient simulation training to improve clinical reasoning: a feasibility randomised controlled trial. BMC Medical Education 2020;20(1):245.

[12] Wade S, Moscova M, Tedla N, et al. Adaptive tutorials versus web-based resources in radiology: a mixed methods analysis of efficacy and engagement in senior medical students. Acad Radiol 2019;26(10):1421-31.

[13] Golshah A, Dehdar F, Imani MM, et al. Efficacy of smartphone-based mobile learning versus lecture-based learning for instruction of cephalometric landmark identification. BMC Med Educ 2020;20(1):287.

[14] Radhika G, Dara AK, Varalaxmi KP, et al. Perceptions of the introduction of objective structured practical examination (OSPE)/objective structured clinical examination (OSCE): a pilot study carried out in Government Medical College, Ananthapuramu, Andhra Pradesh, India. J NTR Univ Health Sci 2015;4(3):145-9. 